\title{
Effective Investment to Reduce Setup Cost in a Mixture Inventory Model Involving Controllable Backorder Rate and Variable Lead Time with a Service Level Constraint
}

\author{
Hsien-Jen Lin \\ Department of Applied Mathematics, Aletheia University, Tamsui, Taipei 25103, Taiwan \\ Correspondence should be addressed to Hsien-Jen Lin, au4409@mail.au.edu.tw
}

Received 5 August 2011; Revised 21 October 2011; Accepted 22 October 2011

Academic Editor: Wanquan Liu

Copyright (c) 2012 Hsien-Jen Lin. This is an open access article distributed under the Creative Commons Attribution License, which permits unrestricted use, distribution, and reproduction in any medium, provided the original work is properly cited.

\begin{abstract}
This paper investigates the impact of setup cost reduction on an inventory policy for a continuous review mixture inventory model involving controllable backorder rate and variable lead time with a service level constraint, in which the order quantity, setup cost, and lead time are decision variables. Our objective is to develop an algorithm to determine the optimal order quantity, setup cost, and lead time simultaneously, so that the total expected annual cost incurred has a minimum value. Furthermore, four numerical examples are provided to illustrate the results, and the effects of system parameters are also included for decision making.
\end{abstract}

\section{Introduction}

Optimal inventory policies have been subject to a lot of research in recent years. In traditional economic order quantity (EOQ) and economic production quantity (EPQ) models, most of the literature treating inventory problems, either in deterministic or probabilistic models, the stockout or setup costs are regarded as prescribed constants and equal at the optimum. However, the experience of the Japanese indicates that this need not be the case. In practice, setup cost may be controlled and reduced by virtue of various efforts, such as worker training, procedural changes, and specialized equipment acquisition. In the literature, Porteus [1] first introduced the concept of investing in reducing the setup cost in the classical EOQ model and determined an optimal setup cost level. The framework he proposed has encouraged many researchers, such as Keller and Noori [2], Nasri et al. [3], Kim et al. [4], Paknejad et al. [5], and Ouyang and Chang [6] to examine setup cost reduction. Moreover, in many inventory problems, the stockout cost is one of the components in the objective function, but, in many 
practical situations, the stockout cost includes intangible components, such as loss of goodwill and potential delay to the other parts of the system, and thus the determination (or estimation) of the stockout cost is considered difficult. Instead of having a stockout cost term in the objective function, a service level constraint, which implies that the stockout level per cycle is bounded, is added to the model. Moreover, a service level criterion is generally easy to interpret and establish. Thus, service level constraint models are more popular in real-life inventory systems than full-cost models (which have generally received far more attention in the theoretical literature). Several researchers (e.g., Aardal et al. [7], Moon and Choi [8], Ouyang and $\mathrm{Wu}$ [9], Chen and Krass [10], Lee et al. [11]) replace the stockout cost by a condition on the service level in order to prevent unacceptable stockouts. We note that these papers focus on inventory models with a service level constraint in which setup cost is treated as a prescribed constant, which is not controlled. Ouyang and Chang [12] considered the setup cost as one of the decision variables, and the backorder rate and the lead time are assumed to be constant. Later, Ouyang et al. [13] considered the setup cost as one of the decision variables and the backorder rate is assumed to be a random variable, which therefore is not subject to control; however, Ouyang and Chuang [14] observed that, under most market behavior as shortages occur, the longer the length of lead time, the larger the amount of shortages, the smaller the proportion of customers who wait, and hence the smaller the backorder rate. In the situation, how to control an appropriate length of lead time to determine a target value of backorder rate so as to minimize the inventory relevant cost and increase the competitive edge is worth discussing. Consequently, we here assume that the backorder rate is dependent on the length of the lead time through the amount of shortages.

Based on the arguments above, we extend the model in [13] and propose a more general model that allows the backorder rate as a control variable and setup cost as a decision variable in conjunction with the order quantity and lead time. We further consider two widely used investment cost functional forms, the logarithmic and the power function, which are consistent with the Japanese experience [15] to analyze the effects of increasing investment to reduce the setup cost. Besides, using the assumptions in [16], lead time can be decomposed into several mutually independent components each having a different crashing cost for shortening lead time. Furthermore, we develop an algorithm to determine the optimal solutions. Finally, numerical examples are presented to illustrate the solution procedure of the proposed model and the effects of the parameters.

The paper is organized as follows: Section 2 details the notation and assumptions. In Section 3, we formulate the controlling setup cost inventory model including a mixture of backorders and lost sales with a service level constraint for what follows, and then two forms of capital investment cost function (logarithmic and power) are developed. Further, an efficient algorithm is developed to find the optimal solutions. In Section 4, four numerical examples are presented to illustrate the solution procedures of the proposed models and the effects of the parameters. The final section concludes the paper.

\section{Notations and Assumptions}

To develop the mathematical model, the following notations are used throughout the paper:

$A$ : setup cost per setup (decision variable)

$A_{0}$ : initial setup cost

$D$ : expected demand per year 
$h$ : inventory holding cost per item per year

$I(A)$ : capital investment required to achieve setup cost $A, 0<A \leq A_{0}$

$L$ : length of lead time (decision variable)

$Q$ : order quantity (decision variable)

$r$ : reorder point

$X$ : the lead time demand which has a normal d.f. $F$ with finite mean $D L$ and standard deviation $\sigma \sqrt{L}$, where $\sigma$ denotes the standard deviation of the demand per year

$\alpha$ : proportion of demands which are not met from stock, that is, $1-\alpha$ is the service level

$\beta$ : fraction of the demand during the stockout period that will be backordered, $\beta \in$ $[0,1]$

$\theta$ : fractional opportunity cost of capital per year

$E(\cdot)$ : mathematical expectation

$z^{+}: z^{+}=z \vee 0$ is the positive part of $z$.

In addition, the following assumptions are made.

(1) The reorder point, $r=$ expected demand during lead time + safety stock (SS), and $\mathrm{SS}=k \times$ (standard deviation of lead time demand), that is, $r=D L+k \sigma \sqrt{L}$, where $k$ is known as the safety factor and satisfies $P(X>r)=q, q$ denotes the allowable stockout probability during the lead time interval.

(2) Inventory is continuously reviewed, and replenishments are made whenever the inventory level falls to the reorder point, $r$.

(3) The lead time $L$ consists of $m$ mutually independent components. The $i$ th component has the normal duration, $b_{i}$, the minimum duration, $a_{i}$, and the crashing cost per unit time, $c_{i}$. Furthermore, these $c_{i}$ are assumed to be arranged such that $c_{1} \leq$ $c_{2} \leq \cdots \leq c_{m}$.

(4) The components of lead time are crashed one at a time starting with the component of least $c_{i}$, and so on.

(5) If we let $L_{i}$ be the length of lead time with components $1,2, \ldots, i$ crashed to their minimum duration, then $L_{\min }=\sum_{i=1}^{m} a_{i} \leq L \leq \sum_{i=1}^{m} b_{i}=L_{\max }, L_{i}=L_{\max }-\sum_{j=1}^{i}\left(b_{j}-\right.$ $\left.a_{j}\right)$, and the lead time crashing cost per cycle $C(L)$ for a given $L \in\left(L_{i}, L_{i-1}\right]$ is given by $C(L)=c_{i}\left(L_{i-1}-L\right)+\sum_{j=1}^{i-1} c_{j}\left(b_{j}-a_{j}\right)$.

(6) During the stockout, the backorder rate, $\beta$, is variable and is a function of $L$ through $E(X-r)^{+}$. The larger the expected shortage quantity, the smaller the backorder rate. Thus, we define that $\beta=\left[1+\xi E(X-r)^{+}\right]^{-1}$, where the backorder parameter, $\xi$, is a positive constant.

(7) The option of investing in reducing setup cost is available. The investment required to reduce the setup cost from initial setup cost $A_{0}$ to a target level $A$ is denoted by $I(A)$, where $I(A)$ is a convex and strictly decreasing function. 


\section{The Basic Models}

In this section we provide a quantitative model for how managers should allocate investments in setup cost reduction programs. For the model without setup cost reduction, we will closely follow the model in [9]. Specifically, the total expected annual cost, which is composed of setup cost, inventory holding cost, and lead time crashing cost, subject to a constraint on service level is expressed as

$$
\begin{aligned}
\operatorname{Min} \operatorname{EAC}(Q, L) & =\frac{A D}{Q}+h\left[\frac{Q}{2}+r-D \mu+(1-\beta) E(X-r)^{+}\right]+\frac{D}{Q} C(L), \\
\text { subject to } & \frac{E(X-r)^{+}}{Q} \leq \alpha,
\end{aligned}
$$

where $E(X-r)^{+}$is the expected number of shortages at the end of the cycle.

According to the opinion of Ouyang and Chuang [14] on the backorder rate (under most market behavior, as shortages occur, the longer the length of lead time, the larger the amount of shortages, the smaller the proportion of customers who wait, and hence the smaller the backorder rate) and in contrast with the model in [9] and further as pointed out by Porteus [1], in the long run, one can allow the setup cost to be a function of capital expenditure; in this section, we consider the backorder rate, $\beta$, as a control variable and the setup cost, $A$, as a decision variable and seek to minimize the total expected annual cost, which is the sum of the capital investment cost of reducing setup cost and the inventory related costs (as expressed in (3.1)) by optimizing over $Q, A$, and $L$, constrained on $0<A \leq A_{0}$ and service level. Mathematically, the problem can be formulated as

$$
\begin{aligned}
& \operatorname{Min} \operatorname{EAC}(Q, A, L)= \theta I(A)+\frac{A D}{Q}+h\left[\frac{Q}{2}+r-D \mu+\left(1-[1+G(L)]^{-1}\right) E(X-r)^{+}\right] \\
&+\frac{D}{Q} C(L), \\
& \text { subject to } \quad 0<A \leq A_{0}, \frac{E(X-r)^{+}}{Q} \leq \alpha,
\end{aligned}
$$

where $G(L)=\xi \sigma \sqrt{L} \Psi(k)$.

We note that the setup cost level is $A \in\left(0, A_{0}\right]$, which implies that if the optimal setup cost obtained does not satisfy the restriction on $A$, then no setup cost reduction investment is made. For this special case, the optimal setup cost is the initial setup cost.

\subsection{Logarithmic Investment Function Case}

In this subsection, we assume that the capital investment, $I(A)$, in reducing setup cost is a logarithmic function of the setup cost $A$. That is, $I(A)=b \ln \left(A_{0} / A\right)$ for $0<A \leq A_{0}$, where $b=$ $1 / \delta$, and $\delta$ is a percentage decreasing in setup cost, $A$, per dollar and increasing in investment $I(A)$. This function is consistent with the Japanese experience [15] and has been used by Porteus [1, 17], Paknejad and Affisco [18], Hong and Hayya [19], Lin [20], and others. 
As mentioned earlier, we have assumed that the lead time demand, $X$, follows a normal distribution with finite mean, $D L$, and standard deviation, $\sigma \sqrt{L}$. We note that $r=$ $D L+k \sigma \sqrt{L}$, and, hence, the expected shortage quantity at the end of the cycle is given by $E(X-r)^{+}=\sigma \sqrt{L} \Psi(k)$, where $\Psi(k)=\phi(k)-k[1-\Phi(k)]$, and $\phi$, $\Phi$ denote the standard normal probability density function and cumulative distribution function, respectively. Therefore, the cost function equation (3.2) can be transformed to

$$
\begin{aligned}
& \operatorname{Min} \operatorname{EAC}^{L}(Q, A, L)= \theta b \ln \left(\frac{A_{0}}{A}\right)+\frac{A D}{Q}+h\left[\frac{Q}{2}+k \sigma \sqrt{L}\right] \\
&+h\left(1-[1+G(L)]^{-1}\right) \sigma \sqrt{L} \Psi(k)+\frac{D}{Q} C(L), \\
& \text { subject to } \quad 0<A \leq A_{0}, \frac{\sigma \sqrt{L} \Psi(k)}{\alpha} \leq Q,
\end{aligned}
$$

where the superscript $L$ in $E A C(\cdot)$ denotes the total expected annual cost for the logarithmic investment function case.

In order to find the minimum cost for this nonlinear programming problem, we first ignore the restriction $0<A \leq A_{0}$ and the service level constraint $\sigma \sqrt{L} \Psi(k) / \alpha \leq Q$ for the moment and minimize the total relevant cost function over $Q, A$, and $L$ with classical optimization techniques by taking the first partial derivatives of $\operatorname{EAC}^{L}(Q, A, L)$ with respect to $Q, A$, and $L \in\left(L_{i}, L_{i-1}\right)$, respectively. We obtain that

$$
\begin{gathered}
\frac{\partial \mathrm{EAC}^{L}(Q, A, L)}{\partial Q}=-\frac{A D}{Q^{2}}+\frac{h}{2}-\frac{D}{Q^{2}} C(L), \\
\frac{\partial \mathrm{EAC}^{L}(Q, A, L)}{\partial A}=-\frac{\theta b}{A}+\frac{D}{Q^{\prime}} \\
\frac{\partial \mathrm{EAC}^{L}(Q, A, L)}{\partial L}=\frac{1}{2} h k \sigma L^{-1 / 2}+\frac{h G^{2}(L)[2+G(L)]}{2 \xi L[1+G(L)]^{2}}-c_{i} \frac{D}{Q} .
\end{gathered}
$$

By examining the second-order sufficient conditions (SOSCs), it can be verified that $\operatorname{EAC}^{L}(Q, A, L)$ is not a convex function of $(Q, A, L)$. However, for fixed $(Q, A), \operatorname{EAC}^{L}(Q, A, L)$ is concave in $L \in\left[L_{i}, L_{i-1}\right]$, since

$$
\frac{\partial^{2} \operatorname{EAC}^{L}(Q, A, L)}{\partial L^{2}}=-\frac{1}{4} h k \sigma L^{-3 / 2}-\frac{h[3+G(L)] G^{3}(L)}{4 \xi L^{2}[1+G(L)]^{3}}<0 .
$$


Thus, for fixed $(Q, A)$, the minimum total expected annual cost will occur at the end points of the interval $\left[L_{i}, L_{i-1}\right]$. Consequently, the problem is reduced to

$$
\begin{aligned}
& \operatorname{Min} \operatorname{EAC}^{L}\left(Q, A, L_{i}\right)= \theta b \ln \left(\frac{A_{0}}{A}\right)+\frac{A D}{Q}+h\left[\frac{Q}{2}+k \sigma \sqrt{L_{i}}\right] \\
&+h\left(1-\left[1+G\left(L_{i}\right)\right]^{-1}\right) \sigma \sqrt{L_{i}} \Psi(k)+\frac{D}{Q} C\left(L_{i}\right), \\
& \text { subject to } \quad 0<A \leq A_{0}, \frac{\sigma \sqrt{L_{i}} \Psi(k)}{\alpha} \leq Q, i=0,1,2, \ldots, m .
\end{aligned}
$$

On the other hand, for a given value of $L \in\left[L_{i}, L_{i-1}\right]$, by solving the equations $\partial \operatorname{EAC}^{L}(Q, A, L) / \partial Q=0$ and $\partial \operatorname{EAC}^{L}(Q, A, L) / \partial A=0$ for $Q$ and $A$, we obtain that

$$
\begin{gathered}
Q^{*}=\left\{\frac{2 D\left[A^{*}+C(L)\right]}{h}\right\}^{1 / 2}, \\
A^{*}=\frac{\theta b Q^{*}}{D}
\end{gathered}
$$

Theoretically, for fixed $L \in\left[L_{i}, L_{i-1}\right]$, from (3.7) and (3.8), we can obtain the values of $Q^{*}$ and $A^{*}$. Moreover, it can be verified that the SOSCs are satisfied as follows. For fixed $L \in\left[L_{i}, L_{i-1}\right]$, let us now consider the Hessian matrix $\mathbf{H}$ as follows:

$$
\mathbf{H}=\left[\begin{array}{ll}
\frac{\partial^{2} \operatorname{EAC}^{L}(Q, A, L)}{\partial Q^{2}} & \frac{\partial^{2} \operatorname{EAC}^{L}(Q, A, L)}{\partial Q \partial A} \\
\frac{\partial^{2} \operatorname{EAC}^{L}(Q, A, L)}{\partial A \partial Q} & \frac{\partial^{2} \operatorname{EAC}^{L}(Q, A, L)}{\partial A^{2}}
\end{array}\right] .
$$

Taking the second partial derivatives of $\operatorname{EAC}^{L}(Q, A, L)$ with respect to $Q$ and $A$, we obtain that

$$
\begin{gathered}
\frac{\partial^{2} \operatorname{EAC}^{L}(Q, A, L)}{\partial Q^{2}}=\frac{2 A D}{Q^{3}}+2 C(L) \frac{D}{Q^{3}}>0 \\
\frac{\partial^{2} \operatorname{EAC}^{L}(Q, A, L)}{\partial Q \partial A}=\frac{\partial^{2} \operatorname{EAC}^{L}(Q, A, L)}{\partial A \partial Q}=-\frac{D}{Q^{2}} \\
\frac{\partial^{2} \operatorname{EAC}^{L}(Q, A, L)}{\partial A^{2}}=\frac{\theta b}{A^{2}} .
\end{gathered}
$$

We proceed by evaluating the principal minor determinant of the Hessian matrix $\mathbf{H}$ at point $\left(Q^{*}, A^{*}\right)$. The first principal minor determinant of $\mathbf{H}$ then becomes

$$
\left|H_{11}\right|=\frac{2 A^{*} D}{Q^{* 3}}+2 C(L) \frac{D}{Q^{* 3}}>0
$$


Next, computing the second principal minor determinant of $\mathbf{H}$ (note that from (3.8), $A^{*}=$ $\left.\theta b Q^{*} / D\right)$, we have

$$
\left|H_{22}\right|=\left(\frac{2 A^{*} D}{Q^{* 3}}+2 C(L) \frac{D}{Q^{* 3}}\right) \frac{\theta b}{A^{* 2}}-\frac{D^{2}}{Q^{* 4}}=\frac{\theta b D}{A^{*} Q^{* 3}}\left[1+\frac{2 C(L)}{A^{*}}\right]>0 .
$$

We conclude that the Hessian matrix $\mathbf{H}$ is positive definite at point $\left(Q^{*}, A^{*}\right)$. Thus, for fixed $L \in\left[L_{i}, L_{i-1}\right]$, the point $\left(Q^{*}, A^{*}\right)$ is the quasioptimal solution (the optimal solution must obey the service level constraint and the restriction on setup cost per setup) so that the total expected annual cost of the logarithmic investment model has a minimum value.

We note that it is not possible to find the closed-form solution for $\left(Q^{*}, A^{*}\right)$ from (3.7) and (3.8); however, the optimal value of $\left(Q^{*}, A^{*}\right)$ can be obtained by adopting a graphical technique similar to that used in [21]. The similar numerical search technique also has been used in $[22,23]$, and others. Thus, we develop the following iterative algorithm to find the optimal values for the order quantity, setup cost, and lead time.

Algorithm 3.1.

Step 1. For each $L_{i}, i=0,1,2, \ldots, m$, and a given $q$ (and hence, the value of safety factor $k$ can be found directly from the standard normal distribution table), perform (i) to (iv).

(i) Start with $A_{i 1}=A_{0}$.

(ii) Substituting $A_{i 1}$ into (3.7) evaluates $Q_{i 1}$.

(iii) Utilizing $Q_{i 1}$ determines $A_{i 2}$ from (3.8).

(iv) Repeat (ii) to (iii) until no change occurs in the values of $Q_{i}$ and $A_{i}$.

Step 2. Compare $A_{i}$ and $A_{0}$.

(i) If $A_{i}<A_{0}$, then $A_{i}$ is feasible and we denote the solution found in Step 1 for given $L_{i}$ by $\left(Q_{L_{i}}, A_{L_{i}}\right)$.

(ii) If $A_{i} \geq A_{0}$, then $A_{i}$ is not feasible and for given $L_{i}$, take $A_{L_{i}}=A_{0}$ and the corresponding value of $Q_{L_{i}}$ can be obtained by substituting $A_{L_{i}}$ into (3.7).

Step 3. Let $x_{i}=\max \left\{Q_{L_{i}},(\sigma / \alpha) \sqrt{L_{i}} \Psi(k)\right\}$.

Step 4 . For each $\left(x_{i}, A_{L_{i}}, L_{i}\right), i=0,1,2, \ldots, m$, compute the corresponding total expected annual cost of the logarithmic investment model $E A C^{L}\left(x_{i}, A_{L_{i}}, L_{i}\right)$, utilizing (3.6).

Step 5. Find $\min _{i=0,1, \ldots, m} \operatorname{EAC}^{L}\left(x_{i}, A_{L_{i}}, L_{i}\right)$.

If $\operatorname{EAC}^{L}\left(Q_{s}, A_{s}, L_{s}\right)=\min _{i=0,1, \ldots, m} \operatorname{EAC}^{L}\left(x_{i}, A_{L_{i}}, L_{i}\right)$, then $\left(Q_{s}, A_{s}, L_{s}\right)$ is the optimal solution. And the optimal backorder rate

$$
\beta_{s}=\frac{1}{1+\xi \sigma \sqrt{L_{s}} \Psi(k)}
$$




\subsection{Power Investment Function}

In contrast to the logarithmic investment function case, in this subsection, we consider the situation where the capital investment, $I(A)$, for reducing setup cost is a power function of the setup cost, $A$. That is,

$$
I(A)=\lambda A^{-\omega}-l, \text { for } 0<A \leq A_{0},
$$

where $l=\lambda A_{0}^{-\omega}$, and $\lambda$ and $\omega$ are positive constants. We note that this particular investment cost function has been used by Porteus [1] and others.

In this case, the cost function (3.2) can be transformed to

$$
\begin{aligned}
& \operatorname{Min} \operatorname{EAC}^{P}(Q, A, L)= \theta\left(\lambda A^{-\omega}-l\right)+\frac{A D}{Q}+h\left[\frac{Q}{2}+k \sigma \sqrt{L}\right] \\
&+h\left(1-[1+G(L)]^{-1}\right) \sigma \sqrt{L} \Psi(k)+\frac{D}{Q} C(L), \\
& \text { subject to } \quad 0<A \leq A_{0}, \frac{\sigma \sqrt{L} \Psi(k)}{\alpha} \leq Q,
\end{aligned}
$$

where the superscript $P$ in $\operatorname{EAC}(\cdot)$ is the total expected annual cost for the power investment function case.

As discussed in the preceding subsection, the problem can be reduced to consider

$$
\begin{aligned}
& \operatorname{Min}_{E^{2}}\left(Q, A, L_{i}\right)= \theta\left(\lambda A^{-\omega}-l\right)+\frac{A D}{Q}+h\left[\frac{Q}{2}+k \sigma \sqrt{L_{i}}\right] \\
&+h\left(1-\left[1+G\left(L_{i}\right)\right]^{-1}\right) \sigma \sqrt{L_{i}} \Psi(k)+\frac{D}{Q} C\left(L_{i}\right), \\
& \text { subject to } \quad 0<A \leq A_{0}, \quad \frac{\sigma \sqrt{L_{i}} \Psi(k)}{\alpha} \leq Q, i=0,1,2, \ldots, m .
\end{aligned}
$$

The solution can be obtained by taking the first partial derivatives of $\operatorname{EAC}^{P}(Q, A, L)$ with respect to $Q$ and $A$, and set them equal to zero, that is, $\partial \operatorname{EAC}^{P}(Q, A, L) / \partial Q=0$ and $\partial \operatorname{EAC}^{P}(Q, A, L) / \partial A=0$. The resulting solutions are

$$
\begin{gathered}
Q=\left\{\frac{2 D[A+C(L)]}{h}\right\}^{1 / 2}, \\
A=\left(\frac{\theta \lambda \omega Q}{D}\right)^{1 /(\omega+1)} .
\end{gathered}
$$

We can apply a similar algorithm as in Section 3.1 to obtain the optimal solution in the power investment function case, in which the optimal values of order quantity, setup cost, lead time, and backorder rate, respectively, are denoted by $\widehat{Q}_{s}, \widehat{A}_{s}, \widehat{L}_{s}$, and $\widehat{\beta}_{s}$. 
Table 1: Lead time data.

\begin{tabular}{lccc}
\hline $\begin{array}{l}\text { Lead time } \\
\text { component, } i\end{array}$ & $\begin{array}{c}\text { Normal duration, } \\
b_{i}(\text { days })\end{array}$ & $\begin{array}{c}\text { Minimum duration, } \\
\left.a_{i} \text { (days }\right)\end{array}$ & $\begin{array}{c}\text { Unit crashing cost, } \\
c_{i}(\$ / \text { day })\end{array}$ \\
\hline 1 & 20 & 6 & 0.4 \\
2 & 20 & 6 & 1.2 \\
3 & 16 & 9 & 5.0 \\
\hline
\end{tabular}

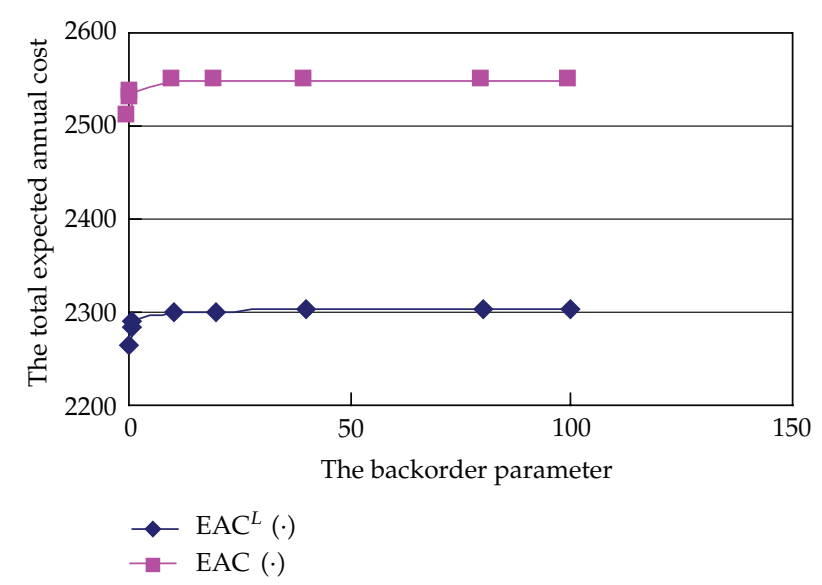

Figure 1: Summary of the results of the optimal procedure for different values of $\xi$. Note that $\operatorname{EAC}^{L}\left(Q_{s}, A_{s}, L_{s}\right)$ and $\operatorname{EAC}\left(Q_{s}, A_{s}, L_{s}\right)$ will be denoted by the symbols $\operatorname{EAC}^{L}(\cdot)$ and $\operatorname{EAC}(\cdot)$, respectively.

\section{Numerical Examples}

Example 4.1. In order to illustrate the above solution procedure and the effects of setup cost reduction, let us consider an inventory system with the following data: $D=600$ units per year, $A_{0}=\$ 200$ per setup, $h=\$ 20$ per unit per year, $\theta=0.1$ per dollar per year, $\sigma=7$ units per week, and the service level $1-\alpha=0.975$; that is, the proportion of demands which are not met from stock is $\alpha=0.025$, and the lead time has three components with data shown in Table 1. Suppose further that the lead time demand follows a normal distribution and the capital investment, $I(A)$, in reducing setup cost can be described by a logarithmic function with the parameter $b=5800$. We want to solve the cases when the backorder parameter $\xi=0,0.5,1,10,20,40,80,100$, and $\infty$ and $q=0.2$ (in this situation, the value of the safety factor, $k$, can be found directly from the standard normal distribution table and is 0.845). Applying the proposed algorithm procedure yields the results shown in Table 2. Furthermore, we list the optimal results of the fixed setup cost model in the same table to illustrate the effects of investing in setup cost reduction (also see Figure 1).

From Table 2, comparing our new model with that of the fixed setup cost case, we observe that the savings range from $9.68 \%$ to $9.83 \%$, which shows that significant savings can be achieved due to controlling the setup cost. Note that the savings and backorder rate $\beta$ increase as $\xi$ decreases. It is also interesting to observe that the optimal order quantity, setup cost, and lead time are the same for various backorder parameter, $\xi$.

Example 4.2. We use the same data as in numerical Example 4.1, and expect that the capital investment $I(A)$ in reducing setup cost is described by a power function with the parameters 


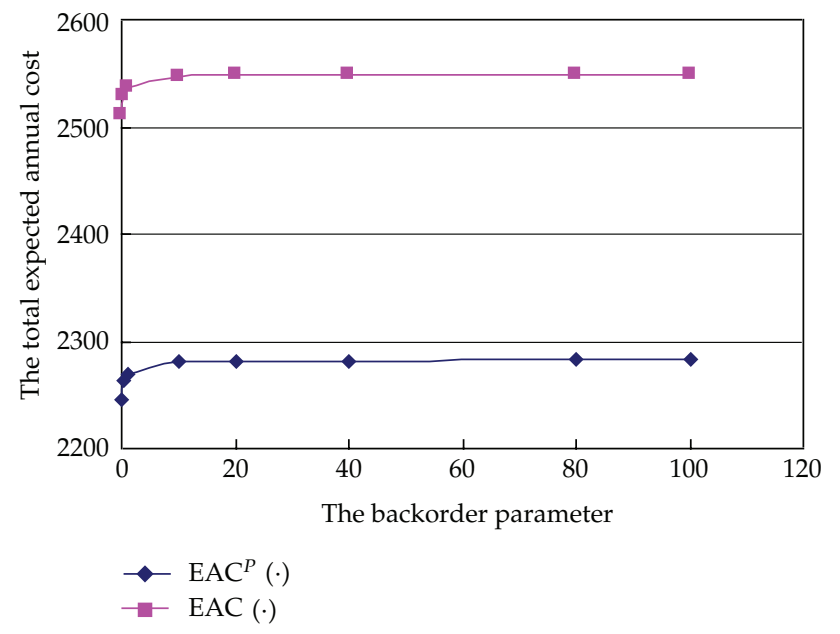

Figure 2: Summary of the results of the optimal procedure for different values of $\xi$. Note that $\operatorname{EAC}^{P}\left(\widehat{A}_{s}, \widehat{Q}_{s}, \widehat{L}_{s}\right)$ and $\operatorname{EAC}\left(\widehat{A}_{s}, \widehat{Q}_{s}, \widehat{L}_{s}\right)$ will be denoted by the symbols $\operatorname{EAC}^{P}(\cdot)$ and $\operatorname{EAC}(\cdot)$, respectively.

Table 2: The optimal solutions for logarithmic investment case in Example 4.1.

\begin{tabular}{lcccccccccc}
\hline \multicolumn{1}{c}{ Setup cost reduction model } \\
$\xi$ & $A_{s}$ & $Q_{s}$ & $L_{s}$ & $\beta_{s}$ & $\operatorname{EAC}^{L}(\cdot)$ & $Q_{s}$ & $L_{s}$ & $\beta_{s}$ & $\operatorname{EAC}(\cdot)$ & Savings $(\%)$ \\
\hline 0.0 & 61 & 76 & 6 & 1 & 2264.29 & 111 & 6 & 1 & 2511.13 & 9.83 \\
0.5 & 61 & 76 & 6 & 0.512 & 2282.84 & 111 & 6 & 0.512 & 2529.68 & 9.76 \\
1.0 & 61 & 76 & 6 & 0.345 & 2289.23 & 111 & 6 & 0.345 & 2536.07 & 9.73 \\
10 & 61 & 76 & 6 & 0.050 & 2300.44 & 111 & 6 & 0.050 & 2547.28 & 9.69 \\
20 & 61 & 76 & 6 & 0.026 & 2301.37 & 111 & 6 & 0.026 & 2548.20 & 9.69 \\
40 & 61 & 76 & 6 & 0.013 & 2301.85 & 111 & 6 & 0.013 & 2548.68 & 9.68 \\
80 & 61 & 76 & 6 & 0.007 & 2302.09 & 111 & 6 & 0.007 & 2548.93 & 9.68 \\
100 & 61 & 76 & 6 & 0.005 & 2302.14 & 111 & 6 & 0.005 & 2548.98 & 9.68 \\
$\infty$ & 61 & 76 & 6 & 0 & 2302.34 & 111 & 6 & 0 & 2549.18 & 9.68 \\
\hline
\end{tabular}

$\lambda=74000$ and $\omega=0.2$. We solve the cases when $\xi=0,0.5,1,10,20,40,80,100$, and $\infty$. Utilizing a similar procedure as proposed in the algorithm, the summarized optimal values are tabulated in Table 3. Furthermore, the optimal results of the no-investment policy are shown in the same table to illustrate the effects of investing in setup cost reduction (also see Figure 2).

The following inferences can be made from the results in Tables 2 and 3.

(1) We observe that adopting different capital investment functions will cause a difference in setup cost. Hence, we have to choose an appropriate capital investment function.

(2) Increasing the value of the backorder parameter, $\xi$, will result in an increase in the total expected annual cost, but a decrease in the backorder rate, $\beta$. Moreover, for different parameter values, $\xi$, the optimal order quantity, setup cost, and lead time are not influenced. 
Table 3: The optimal solutions for power investment case in Example 4.2.

\begin{tabular}{lcccccccccc}
\hline & \multicolumn{4}{c}{ Setup cost reduction model } & \multicolumn{4}{c}{ Fixed setup cost model $(A=200)$} \\
$\xi$ & $\widehat{A}_{s}$ & $\widehat{Q}_{s}$ & $\widehat{L}_{S}$ & $\widehat{\beta}_{S}$ & $\operatorname{EAC}^{P}(\cdot)$ & $Q_{S}$ & $L_{S}$ & $\beta_{S}$ & $\operatorname{EAC}(\cdot)$ & Savings (\%) \\
\hline 0.0 & 71 & 76 & 6 & 1 & 2244.56 & 111 & 6 & 1 & 2511.13 & 10.62 \\
0.5 & 71 & 76 & 6 & 0.512 & 2263.12 & 111 & 6 & 0.512 & 2529.68 & 10.54 \\
1.0 & 71 & 76 & 6 & 0.345 & 2269.51 & 111 & 6 & 0.345 & 2536.07 & 10.51 \\
10 & 71 & 76 & 6 & 0.050 & 2280.72 & 111 & 6 & 0.050 & 2547.28 & 10.46 \\
20 & 71 & 76 & 6 & 0.026 & 2281.64 & 111 & 6 & 0.026 & 2548.20 & 10.46 \\
40 & 71 & 76 & 6 & 0.013 & 2282.12 & 111 & 6 & 0.013 & 2548.68 & 10.46 \\
80 & 71 & 76 & 6 & 0.007 & 2282.37 & 111 & 6 & 0.007 & 2548.93 & 10.46 \\
100 & 71 & 76 & 6 & 0.005 & 2282.42 & 111 & 6 & 0.005 & 2548.98 & 10.46 \\
$\infty$ & 71 & 76 & 6 & 0 & 2282.62 & 111 & 6 & 0 & 2549.18 & 10.46
\end{tabular}

$L_{s}$ in weeks.

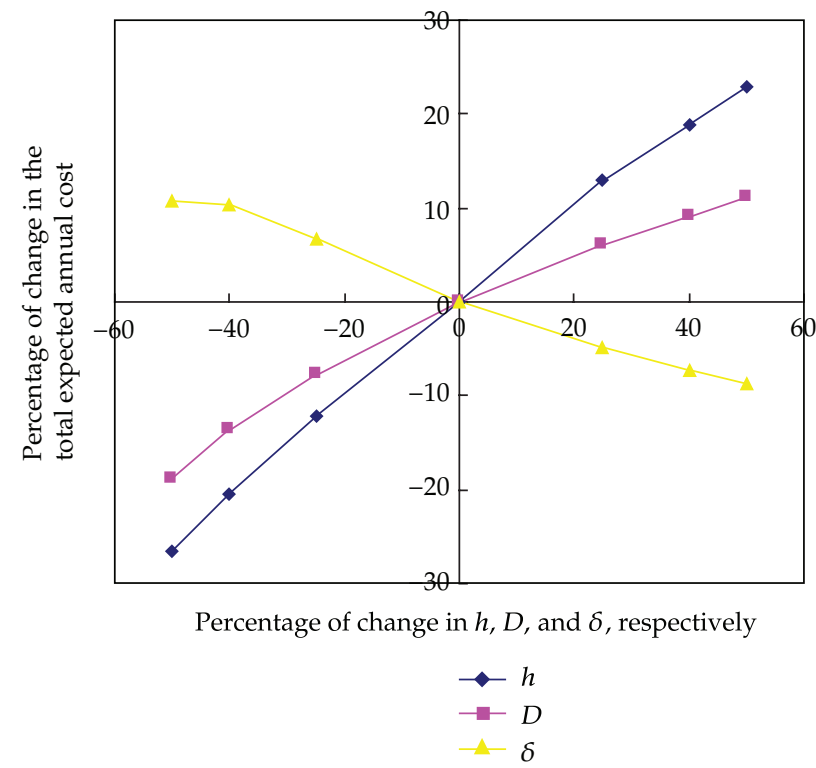

Figure 3: The effects of $h, D$, and $\delta$ on $\operatorname{EAC}^{L}(\cdot)$.

(3) As the value of $\xi$ increases, the total expected annual cost becomes close to the complete lost sales case. Conversely, decreasing the value of $\xi$, the total expected annual cost will approach the complete backorder case.

In addition, we use the logarithmic and power investment functions to examine the effects of changes in the system parameters $h, D$, and $\delta(\lambda, \omega)$ on the optimal order quantity $Q_{s}\left(\widehat{Q}_{s}\right)$, optimal setup cost $A_{S}\left(\widehat{A}_{s}\right)$, optimal lead time $L_{S}\left(\widehat{L}_{s}\right)$, and minimum total expected annual $\operatorname{cost} \operatorname{EAC}^{L}\left(Q_{s}, A_{s}, L_{s}\right)\left(\operatorname{EAC}^{P}\left(\widehat{A}_{s}, \widehat{Q}_{s}, \widehat{L}_{s}\right)\right)$ in Examples 4.1 and 4.2.

Example 4.3. Using the same data and assumptions proposed in Example 4.1, we fix $\xi$ at 0.5 and perform a sensitivity analysis by changing each of the parameters by $+50 \%,+40 \%,+25 \%$, $-25 \%,-40 \%$, and $-50 \%$, taking one parameter at a time and keeping the remaining parameters unchanged. The results are shown in Table 4 and Figure 3. 
Table 4: Effects of change in the parameters for logarithmic investment case in Example 4.1.

\begin{tabular}{|c|c|c|c|c|c|}
\hline \multirow{2}{*}{ Parameters } & \multirow{2}{*}{$\%$ of change } & \multicolumn{3}{|c|}{$\%$ of change in } & \multirow{2}{*}{$L_{s}$} \\
\hline & & $A_{s}$ & $Q_{s}$ & $\operatorname{EAC}^{L}(\cdot)$ & \\
\hline \multirow{6}{*}{$h$} & +50 & -13.11 & -18.42 & +22.89 & 4 \\
\hline & +40 & -8.20 & -18.42 & +18.95 & 4 \\
\hline & +25 & -18.03 & 0 & +13.00 & 6 \\
\hline & -25 & +31.15 & +9.21 & -12.22 & 6 \\
\hline & -40 & +52.46 & +27.63 & -20.45 & 8 \\
\hline & -50 & +83.61 & +52.63 & -26.65 & 8 \\
\hline \multirow{6}{*}{$D$} & +50 & -31.15 & 0 & +11.13 & 6 \\
\hline & +40 & -26.23 & 0 & +9.21 & 6 \\
\hline & +25 & -18.03 & 0 & +6.08 & 6 \\
\hline & -25 & +31.15 & 0 & -7.70 & 6 \\
\hline & -40 & +62.30 & 0 & -13.60 & 6 \\
\hline & -50 & +114.75 & -10.53 & -18.86 & 4 \\
\hline \multirow{6}{*}{$\delta$} & +50 & -50.82 & 0 & -8.64 & 6 \\
\hline & +40 & -45.90 & 0 & -7.21 & 6 \\
\hline & +25 & -32.79 & 0 & -4.82 & 6 \\
\hline & -25 & +72.13 & +6.58 & +6.70 & 6 \\
\hline & -40 & +163.93 & +31.58 & +10.28 & 6 \\
\hline & -50 & +227.87 & +46.05 & +10.81 & 6 \\
\hline
\end{tabular}

$\xi=0.5 ; L_{s}$ in weeks.

On the basis of the results of the Table 4 , the following observations can be made.

(1) $Q_{s}$ and $L_{s}$ decrease while $\operatorname{EAC}^{L}(\cdot)$ increases with an increase in the value of the holding cost parameter, $h$. The results show that $\operatorname{EAC}^{L}(\cdot)$ is moderately sensitive, whereas $Q_{s}$ and $A_{s}$ are highly sensitive to the changes in $h$.

(2) $Q_{s}, L_{s}$, and $\operatorname{EAC}^{L}(\cdot)$ increase, whereas $A_{s}$ decreases with an increase in the value of the demand parameter $D$. Moreover, $Q_{s}$ and $\operatorname{EAC}^{L}(\cdot)$ are moderately sensitive, whereas $A_{s}$ is highly sensitive to the changes in $D$.

(3) $Q_{s}, A_{s}$, and $\operatorname{EAC}^{L}(\cdot)$ decrease with an increase in the value of the model parameter $\delta$. Moreover, $Q_{s}$ and $\operatorname{EAC}^{L}(\cdot)$ are moderately sensitive, whereas $A_{s}$ is highly sensitive to the changes in $\delta$. Besides, we observe that as the value $\delta$ changes, the value $L_{s}$ is not influenced.

Example 4.4. Using the same data and assumptions proposed in Example 4.2, we fix $\xi$ at 0.5 and perform a sensitivity analysis by changing each of the parameters by $+50 \%,+40 \%,+25 \%$, $-25 \%,-40 \%$, and $-50 \%$, taking one parameter at a time and keeping the remaining parameters unchanged. The results are shown in Table 5 and Figure 4. 
Table 5: Effects of change in the parameters for power investment case in Example 4.2.

\begin{tabular}{|c|c|c|c|c|c|}
\hline \multirow{2}{*}{ Parameters } & \multirow{2}{*}{$\%$ of change } & \multicolumn{3}{|c|}{$\%$ of change in } & \multirow{2}{*}{$\widehat{L}_{s}$} \\
\hline & & $\widehat{A}_{S}$ & $\widehat{Q}_{s}$ & $\operatorname{EAC}^{P}(\cdot)$ & \\
\hline \multirow{6}{*}{$h$} & +50 & -11.27 & -18.42 & +23.66 & 4 \\
\hline & +40 & -7.04 & -18.42 & +19.77 & 4 \\
\hline & +25 & -14.08 & 0 & +12.60 & 6 \\
\hline & -25 & +22.54 & +13.16 & -12.21 & 6 \\
\hline & -40 & +36.62 & +30.26 & -20.65 & 8 \\
\hline & -50 & +54.93 & +51.32 & -26.94 & 8 \\
\hline \multirow{6}{*}{$D$} & +50 & -23.94 & 0 & +12.31 & 6 \\
\hline & +40 & -19.72 & 0 & +10.13 & 6 \\
\hline & +25 & -14.08 & 0 & +6.62 & 6 \\
\hline & -25 & +22.54 & 0 & -8.02 & 6 \\
\hline & -40 & +42.25 & 0 & -13.89 & 6 \\
\hline & -50 & +76.06 & -13.16 & -19.01 & 4 \\
\hline \multirow{6}{*}{$\lambda$} & +50 & +76.06 & +15.79 & +8.61 & 6 \\
\hline & +40 & +59.15 & +10.53 & +7.36 & 6 \\
\hline & +25 & +36.62 & +2.63 & +5.02 & 6 \\
\hline & -25 & -32.39 & 0 & -6.06 & 6 \\
\hline & -40 & -49.30 & 0 & -10.46 & 6 \\
\hline & -50 & -59.15 & 0 & -13.88 & 6 \\
\hline \multirow{6}{*}{$\omega$} & +50 & -1.41 & 0 & -1.70 & 6 \\
\hline & +40 & 0 & 0 & -1.11 & 6 \\
\hline & +25 & +1.41 & 0 & -0.42 & 6 \\
\hline & -25 & -9.86 & 0 & -0.98 & 6 \\
\hline & -40 & -22.54 & 0 & -2.65 & 6 \\
\hline & -50 & -33.80 & 0 & -4.46 & 6 \\
\hline
\end{tabular}

$\xi=0.5 ; \widehat{L}_{s}$ in weeks.

On the basis of the results of the Table 5, the following observations can be made.

(1) The results of our computing show that when the power investment function is considered, the optimal values of the order quantity, setup cost, lead time, and total expected annual cost in $h$ and $D$ have the same tendency as in the logarithmic investment function.

(2) $\widehat{Q}_{s}, \widehat{A}_{s}$, and $\operatorname{EAC}^{P}(\cdot)$ increase with an increase in the value of the model parameter $\lambda$. The results show that $\widehat{A}_{s}$ is highly sensitive, whereas $\widehat{Q}_{s}$ and $\operatorname{EAC}^{P}(\cdot)$ are moderately sensitive to the changes in $\lambda$. Besides, we observe that as the value $\lambda$ changes, the value $\widehat{L}_{S}$ is not influenced.

(3) As the value $\omega$ changes, $\widehat{Q}_{s}$ and $\widehat{L}_{s}$ are not influenced. Moreover, $\widehat{A}_{s}$ and $\operatorname{EAC}^{L}(\cdot)$ are moderately sensitive to the changes in $\omega$.

\section{Concluding Remarks}

The purpose of this paper is to investigate a mixture inventory policy on a controlling setup cost in the stochastic continuous review model involving controllable backorder rate and 


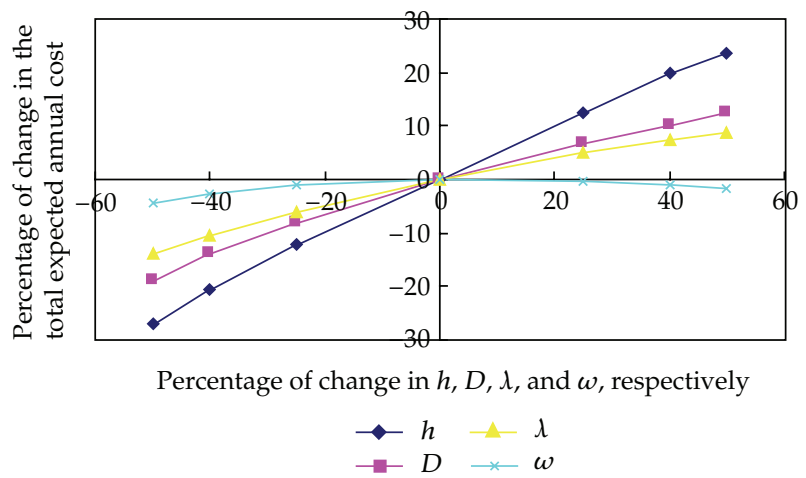

Figure 4: The effects of $h, D, \lambda$, and $\omega$ on $\operatorname{EAC}^{P}(\cdot)$.

variable lead time in which the stockout cost is replaced with a service level constraint that requires a certain level of service to be met in every cycle. We consider two forms of commonly used investment cost functions, logarithmic and power, to reduce setup cost. By analyzing the total expected annual cost, we develop an algorithm to determine the optimal order quantity, setup cost, and lead time so that the total expected annual cost incurred has the minimum value. The results of the numerical examples indicate that if we make decisions with capital investment in reducing setup cost, it would help to lower the system cost, and we can obtain a significant amount of savings. To understand the effects of the optimal solution on changes in the value of the different parameters associated with the inventory system, sensitivity analysis is performed. Furthermore, we observe from the sensitivity analysis that there are slight differences between the two capital investment functions. From Table 4, we see that the optimal setup cost and the total expected annual cost decrease with an increased parameter $\delta$ for the logarithmic functions. Nevertheless, from Table 5, the optimal setup cost and the total expected annual cost increase with an increased parameter $\lambda$ for the power function.

\section{Acknowledgments}

The author would like to thank the editor and the referees for their helpful comments and suggestions.

\section{References}

[1] E. L. Porteus, "Investing in reduction setups in the EOQ model," Management Science, vol. 31, no. 8, pp. 998-1010, 1985.

[2] G. Keller and H. Noori, "Justifying new technology acquisition through its impact on the cost of running an inventory policy," Institute of Industrial Engineers Transactions, vol. 20, no. 3, pp. 284-291, 1988.

[3] F. Nasri, J. F. Affisco, and M. J. Paknejad, "Setup cost reduction in an inventory model with finiterange stochastic lead times," International Journal of Production Research, vol. 28, no. 1, pp. 199-212, 1990.

[4] S. L. Kim, J. C. Hayya, and J. D. Hong, "Setup reduction in economic production quantity model," Decision Sciences, vol. 23, no. 2, pp. 500-508, 1992.

[5] M. J. Paknejad, F. Nasri, and J. F. Affisco, "Defective units in a continuous review $(s, Q)$ system," International Journal of Production Research, vol. 33, no. 10, pp. 2767-2777, 1995. 
[6] L. Y. Ouyang and H. C. Chang, "Lead time and ordering cost reductions in continuous review inventory systems with partial backorders," Journal of the Operational Research Society, vol. 50, no. 12, pp. 1272-1279, 1999.

[7] K. Aardal, Ö. Jonsson, and H. Jönsson, "Optimal inventory policies with service-level constraints," Journal of the Operational Research Society, vol. 40, no. 1, pp. 65-73, 1989.

[8] I. Moon and S. Choi, "The distribution free continuous review inventory system with a service level constraint," Computers and Industrial Engineering, vol. 27, no. 1-4, pp. 209-212, 1994.

[9] L. Y. Ouyang and K. S. Wu, "Mixture inventory model involving variable lead time with a service level constraint," Computers E Operations Research, vol. 24, no. 9, pp. 875-882, 1997.

[10] F. Y. Chen and D. Krass, "Inventory models with minimal service level constraints," European Journal of Operational Research, vol. 134, no. 1, pp. 120-140, 2001.

[11] W. C. Lee, J. W. Wu, and J. W. Hsu, “Computational algorithm for inventory model with a service level constraint, lead time demand with the mixture of distributions and controllable negative exponential backorder rate," Applied Mathematics and Computation, vol. 175, no. 2, pp. 1125-1138, 2006.

[12] L. Y. Ouyang and H. C. Chang, "Mixture inventory model involving setup cost reduction with a service level constraint," Journal of the Operational Research Society of India, vol. 37, no. 4, pp. 327-339, 2001.

[13] L. Y. Ouyang, B. R. Chuang, and H. C. Chang, "Setup cost and lead time reductions on stochastic inventory models with a service level constraint," Journal of the Operations Research Society of Japan, vol. 45, no. 2, pp. 113-122, 2002.

[14] L. Y. Ouyang and B. R. Chuang, "Mixture inventory model involving variable lead time and controllable backorder rate," Computers and Industrial Engineering, vol. 40, no. 4, pp. 339-348, 2001.

[15] R. W. Hall, Zero Inventories, Dow Jones-Irwin, Homewood, Ill, USA, 1983.

[16] C. J. Liao and C. H. Shyu, "An analytical determination of lead time with normal demand," International Journal of Operations \& Production Management, vol. 11, no. 9, pp. 72-78, 1991.

[17] E. L. Porteus, "Optimal lot sizing, process quality improvement and setup cost reduction," Operations Research, vol. 34, no. 1, pp. 137-144, 1986.

[18] M. J. Paknejad and J. F. Affisco, "The effect of investment in new technology on optimal batch quantity," Proceedings of the Northeast Decision Sciences Institute, pp. 118-120, 1987.

[19] J. D. Hong and J. C. Hayya, "Joint investment in quality improvement and setup reduction," Computers and Operations Research, vol. 22, no. 6, pp. 567-574, 1995.

[20] Y. J. Lin, "An integrated vendor-buyer inventory model with backorder price discount and effective investment to reduce ordering cost," Computers and Industrial Engineering, vol. 56, no. 4, pp. 1597-1606, 2009.

[21] G. Hadley and T. M. Whitin, Analysis of Inventory Systems, Prentice-Hall, Englewood Cliffs, NJ, USA, 1963.

[22] I. Moon and S. Choi, "A note on lead time and distributional assumptions in continuous review inventory models," Computers $\mathcal{E}$ Operations Research, vol. 25, no. 11, pp. 1007-1012, 1998.

[23] L. Y. Ouyang, K. S. Wu, and C. H. Ho, "Integrated vendor-buyer cooperative models with stochastic demand in controllable lead time," International Journal of Production Economics, vol. 92, no. 3, pp. 255-266, 2004. 


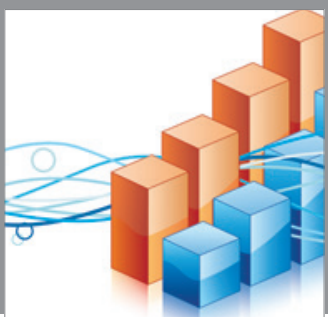

Advances in

Operations Research

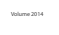

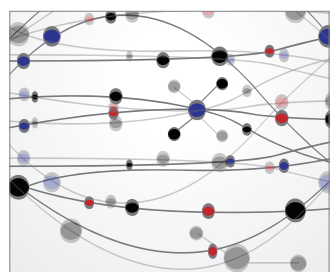

\section{The Scientific} World Journal
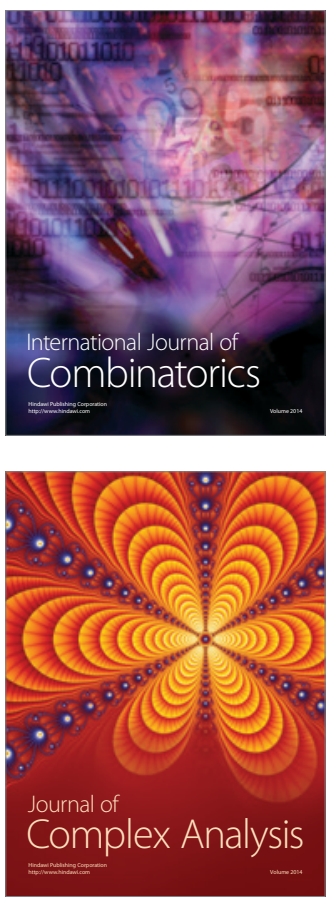

International Journal of

Mathematics and

Mathematical

Sciences
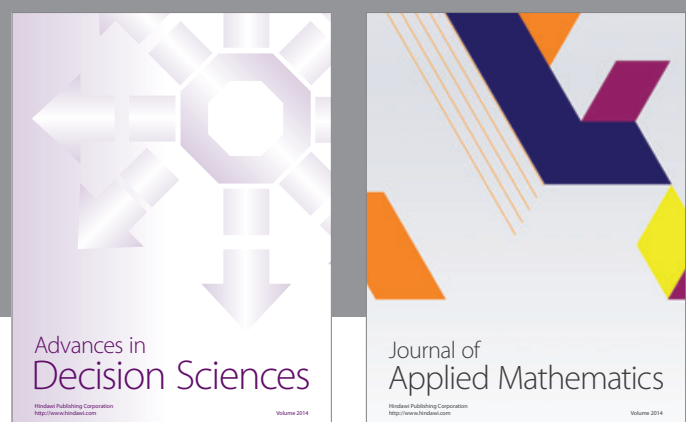

Journal of

Applied Mathematics
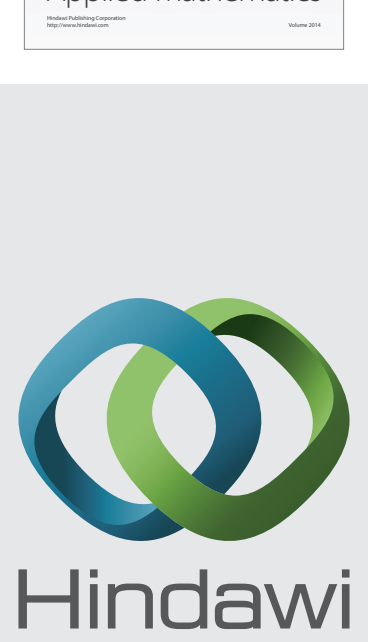

Submit your manuscripts at http://www.hindawi.com
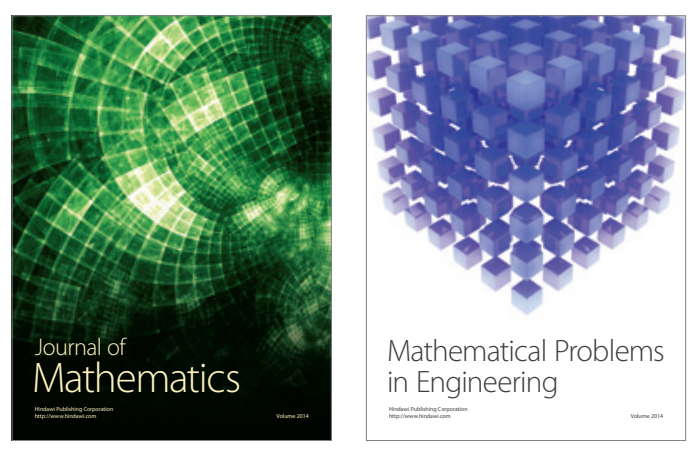

Mathematical Problems in Engineering
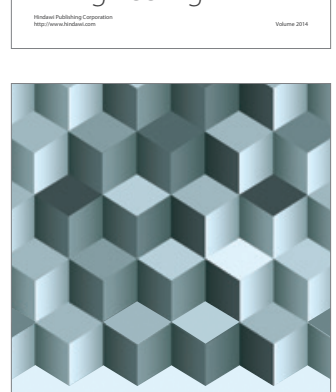

Journal of

Function Spaces
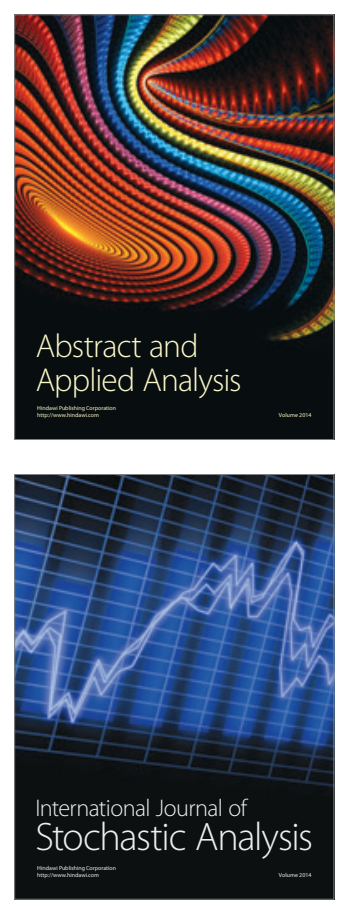

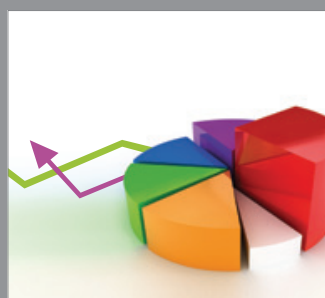

ournal of

Probability and Statistics

Promensencen
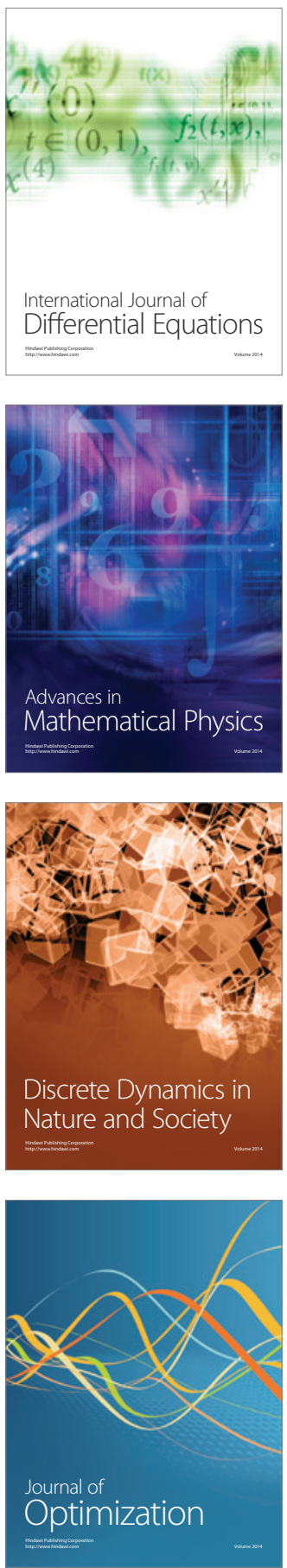